\title{
A new duality transformation for fourth-order gravity
}

\author{
Hans-Jürgen Schmidt \\ University Potsdam, Institute for Mathematics, Cosmology group \\ D-14415 POTSDAM, PF 601553, Am Neuen Palais 10, Germany \\ e-mail hjschmi@rz.uni-potsdam.de
}

\begin{abstract}
We prove that for non-linear $L=L(R), G=d L / d R \neq 0$ the Lagrangians $L$ and $\hat{L}(\hat{R})$ with $\hat{L}=2 R / G^{3}-3 L / G^{4}, \hat{g}_{i j}=G^{2} g_{i j}$ and $\hat{R}=3 R / G^{2}-4 L / G^{3}$ give conformally equivalent fourth-order field equations being dual to each other. The proof represents a new application of the fact that the operator $\square-\frac{R}{6}$ is conformally invariant.
\end{abstract}

Gen. Rel. Grav. in print;

KEY: Conformal relations in fourth-order gravity

\section{Introduction}

Higher-order theories, especially fourth-order gravity theories, are subject to conflicting facts: On the one hand, they appear quite naturally from generally accepted principles; on the other hand, they are unstable and so, they should be considered unphysical.

Nevertheless, from time to time one can find a statement like: "Whichever turns out to be the real theory of gravitation, the corresponding low-energy effective Lagrangian will probably contain higher derivative terms." [1], so it makes sense to elucidate the structure of such theories without specifying a concrete physical context. 
In sct. 2, we shortly review some classes of conformally related theories, especially, to show the difference to our new approach of sct. 3 .

Sct. 3 deals with fourth-order gravity following from a non-linear Lagrangian $L(R)$. The conformal equivalence of these theories to theories of other types is widely known, but the conformal equivalence of these theories to theories of the same type but with different Lagrangian is much less known. We fill this gap by proving a duality theorem between pairs of such fourth-order theories.

\section{Conformally related theories}

In Rainich (1925, ref. [2]) the electromagnetic field was calculated from the curvature tensor. This was cited in Kuchař (1963, ref. [3]) as example for the geometrization programme; in [3] on meson fields $\psi$ (now called scalar fields), Kuchař gives a kind of geometrization by using a relation between $\psi$ and $R$, then he gets the equation

$$
\square R-\frac{k^{2}}{2} R=0
$$

which is of fourth order in the metric. This is the trace of fourth-order gravity as we are dealt with, but he did not deduce it from a curvature squared action.

In Bekenstein (1974, ref. [4]) the conformal transformation from Einstein's theory with a minimally coupled $(\phi)$ to a conformally coupled $(\psi)$ scalar field is proven where additional conformally invariant matter (radiation) is allowed. For $8 \pi G=1$ one has

$$
\psi=\sqrt{6} \tanh (\phi / \sqrt{6})
$$

If radiation is absent and $\phi \neq 0$ then it works also with "coth" instead of "tanh". This is reformulated in his theorem 2: If $g_{i j}$ and $\psi$ form an Einsteinconformal scalar solution with $\psi^{2} \neq 6$, then $\hat{g}_{i j}=\frac{1}{6} \psi^{2} g_{i j}$ and $\hat{\psi}=6 / \psi$ form a second one. One can see that this is a dual map because by applying the operator ^ twice, the original solution is re-obtained. 
Let us comment this theorem 2: For the conformal scalar field one has the effective gravitational constant $G_{\text {eff }}$ defined by

$$
\frac{1}{8 \pi G_{\text {eff }}}=1-\frac{\psi^{2}}{6}
$$

A positive value $G_{\text {eff }}$ implies a negative value $\hat{G}_{\text {eff }}$ and vice versa. By changing the overall sign of the Lagrangian one can achieve a positive effective gravitational constant at the price of the scalar field becoming a ghost (wrong sign in front of the kinetic term). So, Bekenstein has given a conformal transformation from Einstein's theory with a conformally coupled ordinary scalar field to Einstein's theory with a conformally coupled ghost. From this property one can see that this duality relation is different from the duality theorem to be deduced at in sct. 3, because there are no ghosts at all.

Later but independently of [4] the conformal equivalence between minimally and conformally coupled scalar fields with $G_{\text {eff }}>0$ was generalized in [5] by the inclusion of several self-interaction terms. Cf. also ref. [6] for further generalizations to arbitrary coupling parameter $\xi$ and higher dimensions.

The conformal transformation from fourth-order gravity to Einstein's theory with a minimally coupled scalar field was deduced in several steps: Bicknell (1974, ref. [7]) found the transformation for $L=\frac{1}{2} R^{2}$; the conformal factor is $|R|$, and after the transformation, which is valid for $R \neq 0$, one gets Einstein's theory with non-vanishing $\Lambda$-term and a massless minimally coupled scalar field as source.

Similar results for $L(R)$ and conformal factor $\left|\frac{d L}{d R}\right|$ have been deduced in [8], where $\frac{d L}{d R} \neq 0$ and

$$
\frac{d^{2} L}{d R^{2}} \neq 0
$$

have to be fulfilled. The second paper of ref. [8] generalized the conformal transformation to more general tranformations of the metric. Later on and in a different context (metric-affine theories) such transformations have been considered also by Jakubiec and Kijowski [9]. Ref. [10] generalized these conformal transformations by the inclusion of non-minimally coupled scalar fields. In [6] it is noted that it may happen that one singular and another non-singular model may be conformally related. Magnano and Sokołowski 
[11] discuss which of the conformally related frames can be considered to be physical.

\section{A duality theorem}

Let us now deduce the duality theorem announced in the introduction which shall close a gap in the set of the aforementioned results. Two predecessors exist already:

Buchdahl (1978, ref. [12]) showed: For $L=\frac{1}{2} R^{2}$ the conformal factor $R^{2}$ (if it is $\neq 0$ ) transforms solutions to solutions and represents a dual map in the set of solutions. (Another conformal factor than in ref. [7], cf. sct. 2).

Ref. [13] generalizes this dual map to other non-linear Lagrangians $L(R)=\frac{1}{k+1} R^{k+1}, \quad($ where $k \neq-1,0)$, the conformal factor being $R^{2 k}$, and $R$ is supposed to be different from zero. (Again, this conformal factor is the square of that conformal factor which is necessary to transform to Einstein's theory with a minimally coupled scalar field.)

\subsection{The general 4-dimensional case}

Let us now start with the key element of the deduction. For a metric $g_{i j}$ and a scalar $G \neq 0$ we define the conformally related metric

$$
\hat{g}_{i j}=G^{2} g_{i j}
$$

Our strategy is to develop the hat $\wedge$ to a duality operator: Every valid statement shall remain correct if all hats are removed and all formerly unhatted quantities get a hat. For eq. (1) this means $g_{i j}=\hat{G}^{2} \hat{g}_{i j}$. So duality requires

$$
G \hat{G}=1
$$

implying $\hat{G} \neq 0$. For

$$
\square_{c} \equiv \square-\frac{R}{6}
$$

the validity of

$$
G^{3}{ }^{\wedge} \square_{c}=\square_{c} G
$$


reflects the conformal invariance of the operator $\square_{c}$ if applied to any scalar $\chi$. Now we apply eq. (3) to the constant scalar $\chi=-6$ and get

$$
G^{3} \hat{R}=G R-6 \square G
$$

Duality implies

$$
\hat{G}^{3} R=\hat{G} \hat{R}-6^{\wedge} \square \hat{G}
$$

Now we are prepared to consider a gravitational Lagrangian $L=L(R)$ where $L$ is a smooth function (or, at least $C^{3}$-differentiable). We define $G=\frac{d L}{d R}$ and $H=\frac{d^{2} L}{d R^{2}}$ and restrict to an $R$-interval where $G H \neq 0$. Then $L$ gives rise to a fourth-order field equation. We decompose this equation into the trace

$$
3 \square G=2 L-G R
$$

and the trace-free part. The latter is equivalent to require that the trace-free part of the tensor $G R_{i j}-G_{; i j}$ vanishes. We insert eq. (6) into eq. (4) and get

$$
\hat{R}=\frac{3 R}{G^{2}}-\frac{4 L}{G^{3}}
$$

The dual to eq. (7) reads

$$
R=\frac{3 \hat{R}}{\hat{G}^{2}}-\frac{4 \hat{L}}{\hat{G}^{3}}
$$

For $\hat{R}$ we insert the expression (7), for $\hat{G}$ we use eq. (2), and then we can solve eq. (8) for $\hat{L}$ as follows

$$
\hat{L}=\frac{2 R}{G^{3}}-\frac{3 L}{G^{4}}
$$

Applying $\frac{d}{d R}$ to eq. (7) we get

$$
\frac{d \hat{R}}{d R}=\frac{6 H}{G^{4}}(2 L-G R)-\frac{1}{G^{2}}
$$

with the consequence that $\frac{d \hat{R}}{d R} \neq 0$ if and only if

$$
G^{2} \neq 6 H(2 L-G R)
$$

Let (11) be fulfilled in the following, then eq. (7) can be locally inverted as $R=R(\hat{R})$ and with $(9)$ we get $\hat{L}=\hat{L}(\hat{R})$. It is useful to make the following consistency test: Calculate

$$
\hat{G}=\frac{d \hat{L}}{d \hat{R}}=\frac{d \hat{L}}{d R} \cdot\left(\frac{d \hat{R}}{d R}\right)^{-1}
$$


via eqs. (9), (10); one gets $\hat{G}=\frac{1}{G}$ consistent with eq. (2). The analogous consistency takes place with the fourth-order field equation. This proves the following

Theorem: Let $g_{i j}$ be a solution of the field equation following from $L(R)$ then $\hat{g}_{i j}$ is a solution for $\hat{L}(\hat{R})$.

The duality theorem deduced above is a method to construct new solutions of fourth-order gravity from known solutions of a (possibly other) fourth-order theory.

This theorem is most powerful if applied to solutions with non-constant value $R$; the reason is obvious: On the one hand, the solutions with constant $R$ are identical to solutions of Einstein's vacuum equation with suitably chosen cosmological term, and, on the other hand, the conformal factor is constant for this case.

If we replace $L(R)$ by $c \cdot L(R)$ then $\hat{L}(\hat{R})$ is changed to $\hat{L}\left(\hat{R} / c^{2}\right) / c^{3}$. So, up to a scale-transformation, nothing is changed.

$L$ and $\hat{L}$ represent the same function if and only if the corresponding potential $V(\Phi)$ is an even function in $\Phi$ (in that conformal picture where Einstein's theory with the minimally coupled scalar field $\Phi$ and potential $V$ is applied, cf. refs. [8]).

\subsection{Special examples}

For $L=\frac{1}{2} R^{2}$ we get $G=R, \hat{g}_{i j}=R^{2} g_{i j}$, i.e., only the range $R \neq 0$ is allowed. The trace eq. (6) reduces to $\square R=0$; and eq. (4) can be written as

$$
\hat{R}=\frac{1}{R}-\frac{6}{R} \square R
$$

an identity which might be useful in another context, too; it has the following corollary: Let $R \neq 0$ and $\hat{g}_{i j}=R^{2} g_{i j}$. Then the following 3 equations are equivalent:

$$
\square R=0, \quad{ }^{\natural} \square \hat{R}=0, \quad R \hat{R}=1
$$

Now let $L=\frac{1}{k+1}|R|^{k+1}$ with $R \neq 0$ and $k \neq-1,0 . \quad k=1$ leads to the above case. We have $G= \pm|R|^{k}$ where the lower sign corresponds to the case $R<0$. We get

$$
\hat{g}_{i j}=|R|^{2 k} g_{i j}
$$


and

$$
\hat{R}=\frac{3 k-1}{k+1} \cdot \frac{R}{|R|^{2 k}}
$$

Requirement (11) reads $1 \neq 6 k \cdot \frac{1-k}{1+k}$ and implies $k \neq \frac{1}{3}, \frac{1}{2}$. We get

$$
\hat{L}=\frac{2 k-1}{k+1} \cdot \frac{|R|}{|R|^{3 k}}=\hat{c} \cdot|\hat{R}|^{\hat{k}+1}
$$

with $\hat{k}=\frac{1}{2-1 / k}$ and a certain $\hat{c}(k) \neq 0$. The restrictions $k \neq-1,0$ are immediately clear because $L(R)$ has to be nonlinear in $R$. To elucidate the restrictions $k \neq \frac{1}{3}, \frac{1}{2}$ we rewrite eqs. (7)/(9) as follows which is valid in the range $R>0$ ( $R<0$ is quite similar to deal with)

$$
\hat{R}=\frac{3 R^{7 / 3}}{G^{3}} \frac{d}{d R}\left(\frac{L}{R^{4 / 3}}\right)
$$

and

$$
\hat{L}=\frac{2 R^{5 / 2}}{G^{4}} \frac{d}{d R}\left(\frac{L}{R^{3 / 2}}\right)
$$

So, for $k=\frac{1}{3}$ we get $\hat{R} \equiv 0$, for $k=\frac{1}{2}$ we get $\hat{L} \equiv 0$.

A new example reads as follows: Let $L=\frac{1}{4}\left(3 R^{4 / 3}-1\right)$, and let $R>0$. We get $G=R^{1 / 3}, \hat{R}=\frac{1}{R}$, and $\hat{L}(\hat{R})$ is the same function as $L(R)$. So it holds: If $g_{i j}$ is a solution of the corresponding field equation with $R>0$ then $R^{2 / 3} g_{i j}$ is a solution, too. (In the conformal picture with Einstein's theory this example corresponds to a potential $V(\Phi)$ which is a multiple of $\cosh (\sqrt{8 / 3} \Phi)$.)

\subsection{Generalization to higher order and higher dimen- sion}

In [14], the conformal invariance of the operator $\mathbf{D}$ defined by

$$
\mathbf{D}=\square^{2}+2 R^{i j} \nabla_{i} \nabla_{j}-\frac{2}{3} R \square+\frac{1}{3} R^{; i} \nabla_{i}
$$

in four dimensions was shown. More detailed: Let $\hat{g}_{i j}=G^{2} g_{i j}$ then

$$
G^{4} \hat{\mathbf{D}}=\mathbf{D}
$$

if applied to arbitrary scalars. It would be interesting to look whether this identity has similar consequences as eq. (3). 
Up to now, we have considered the 4-dimensional case only. Let us give the corresponding result for an arbitrary dimension $n \geq 3$. We get

$$
\square_{c} \equiv \square-\xi R
$$

where $\xi=\frac{n-2}{4(n-1)}$, and we follow the route sketched in ref. [15], sct. 3 and in Maeda ref. [10]. We replace eq. (1) by

$$
\hat{g}_{i j}=G^{4 /(n-2)} g_{i j}
$$

for any non-vanishing scalar $G$. Without loss of generality we may assume $G>0$ (for, otherwise, we might replace $L$ by $-L$ ), and we keep $G=\frac{d L}{d R}$, $H=\frac{d^{2} L}{d R^{2}} \neq 0, \hat{G}=1 / G$. The trace-free part is again equivalent to require that the trace-free part of the tensor $G R_{i j}-G_{; i j}$ vanishes. However, the trace of the field equation following from $L(R)$ changes and is equivalent to

$$
(n-1) \square G=\frac{n}{2} L-G R
$$

in place of eq. (6). Eq. (3) has to be replaced by (cf. e. g. [6] to see how to calculate the necessary powers of $G$ )

$$
G^{(n+2) /(n-2) \wedge} \square_{c}=\square_{c} G
$$

We apply eq. (16) to the constant scalar $-1 / \xi$ and get instead of eq. (4) now

$$
G^{(n+2) /(n-2)} \hat{R}=G R-\frac{1}{\xi} \square G
$$

We insert eq. (15) into eq. (17) and get

$$
\hat{R}=G^{-(n+2) /(n-2)}\left(\frac{n+2}{n-2} R G-\frac{2 n}{n-2} L\right)
$$

in place of eq. (7). The dual to eq. (18) reads

$$
R=\hat{G}^{-(n+2) /(n-2)}\left(\frac{n+2}{n-2} \hat{R} \hat{G}-\frac{2 n}{n-2} \hat{L}\right)
$$

and so we get in place of eq. (9) now

$$
\hat{L}=G^{-(n+2) /(n-2)}\left(\frac{4}{n-2} R-\frac{n+2}{n-2} \cdot \frac{L}{G}\right)
$$


Applying $\frac{d}{d R}$ to eq. (18) we get

$$
\frac{d \hat{R}}{d R}=G^{-(n+2) /(n-2)}\left[\frac{H(n+2)}{(n-2)^{2}}\left(\frac{2 n L}{G}-4 R\right)-G\right]
$$

So we suppose the r.h.s. of eq. (21) to be unequal zero. With these conditions the theorem of sct. 3.1. keeps valid.

\section{Discussion}

The duality operator was introduced in sct. 3.1 to simplify the deduction of a fourth-order gravity result, however, it seems to be applicable to other situations, too.

Let us give a non-trivial application for fourth-order gravity (it is the last example of subsection 3.2 . but now restricted to the range $R<0$ ). We have

$$
L=\frac{3}{4}(-R)^{4 / 3}-\frac{1}{4}
$$

$\hat{R}=1 / R$, and $\hat{L}$ coincides with $L$. To find out all solutions with $R<$ 0 following from the corresponding field equation it suffices to determine all solutions fulfilling $-1 \leq R<0$. The duality $\hat{g}_{i j}=(-R)^{2 / 3} g_{i j}$ then gives rise to all the solutions fulfilling $R \leq-1$, and they may be matched together smoothly at the hypersurface $R=-1$. So, e.g. the behaviour near a singularity $R \longrightarrow-\infty$ can be studied by considering the solutions in the finite $R$-interval $-1 \leq R<0$. Finally, it might be interesting to observe that the de Sitter space-time with Hubble parameter $h=1 /(2 \sqrt{3})$, i.e., with $R=-1$ is an attractor solution for this field equation (at least within the set of spatially flat Friedman models.)

Acknowledgement. I thank Dr. U. Kasper, Dr. M. Rainer and one of the referees for making some clarifying remarks. Financial support from the Wissenschaftler-Integrations-Programm and from the Deutsche Forschungsgemeinschaft is gratefully acknowledged.

\section{References}

[1] Dobado, A. and Maroto, A. (1995) Phys. Rev. D 52, 1895. 
[2] Rainich, G. (1925) Trans. Amer. Math. Soc. (TAMS) 27, 106.

[3] Kuchař, K. (1963) Czech. J. Phys. B 13, 551.

[4] Bekenstein, J. (1974) Ann. Phys. NY 82, 535.

[5] Deser, S. (1984) Phys. Lett. B 134, 419; Schmidt, H.-J. (1988) Phys. Lett. B 214, 519.

[6] Rainer, M. (1995) Int. J. Mod. Phys. D 4, 397; Rainer, M. (1995) Gravitation and Cosmology 1, 121.

[7] Bicknell, G. (1974) J. Phys. A 7, 1061.

[8] Whitt, B. (1984) Phys. Lett. B 145, 176; Magnano, G., Ferraris, M. and Francaviglia, M. (1987) Gen. Relat. Grav. 19, 465; Schmidt, H.-J. (1987) Astron. Nachr. 308, 183; Barrow, J. and Cotsakis, S. (1988) Phys. Lett. B $\mathbf{2 1 4}, 515$.

[9] Jakubiec, A. and Kijowski, J. (1988) Phys. Rev. D 37, 1406; Jakubiec, A. and Kijowski, J. (1989) J. Math. Phys. 30, 1073; Jakubiec, A. and Kijowski, J. (1989) J. Math. Phys. 30, 2923.

[10 Maeda, K. (1989) Phys. Rev. D 39, 3159; Amendola, L., Litterio, M. and Occhionero, F. (1990) Int. J. Mod. Phys. A 5, 3861.

[11] Magnano, G. and Sokołowski, L. (1994) Phys. Rev. D 50, 5039.

[12] Buchdahl, H. (1978) Int. J. theor. Phys. 17, 149.

[13] Schmidt, H.-J. (1989) Class. Quant. Grav. 6, 557.

[14] Elizalde, E. and Shapiro, I. (1995) Class. Quant. Grav. 12, 1385.

[15] Schmidt, H.-J. (1988) Astron. Nachr. 309, 307. 